\title{
November 2016 Imaging Case of the Month
}

\author{
Michael B. Gotway, MD \\ Department of Radiology \\ Mayo Clinic Arizona \\ Scottsdale, Arizona USA
}

Clinical History: A 38-year-old man presented to his primary care physician with complaints of pruritus, jaundice, and poor appetite. The patient had been diagnosed with hypertension one year earlier and was treated with hydrochlorothiazide and an angiotensin-converting enzyme inhibitor, but evidently did not tolerate the regimen well, and developed "tea-colored" urine following initiation of this therapy. He was also recently diagnosed with diabetes mellitus and also complained of intermittent right upper quadrant pain.

Laboratory data, including white blood cell count and serum chemistries were within normal limits. Oxygen saturation on room air was $99 \%$.

Frontal and lateral chest radiographs (Figure 1) were performed.

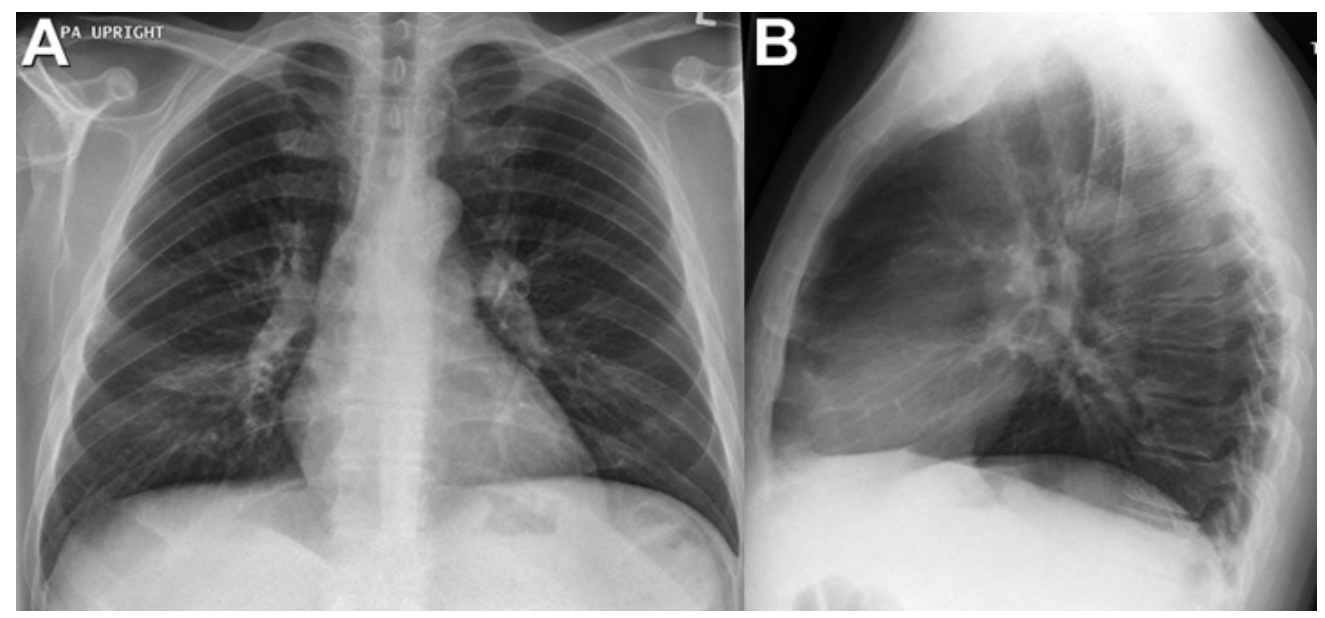

Figure 1. Frontal (A) and lateral (B) chest radiography.

Which of the following statements regarding the chest radiograph is most accurate?

1. Frontal and lateral chest radiography appears normal

2. Frontal and lateral chest radiography shows a focal, poorly defined right base opacity

3. Frontal and lateral chest radiography shows bilateral peribronchial and mediastinal lymph node enlargement

4. Frontal and lateral chest radiography shows cardiomegaly

5. Frontal and lateral chest radiography shows upper lobe bilateral linear and reticular abnormalities 


\section{Correct! \\ 2. Frontal and lateral chest radiography shows a focal, poorly defined right base opacity}

Frontal and lateral chest radiograph shows a focal, somewhat oblong and poorly defined right base opacity, seen on the frontal projection, but not well visualized on the lateral projection (Figure 2).

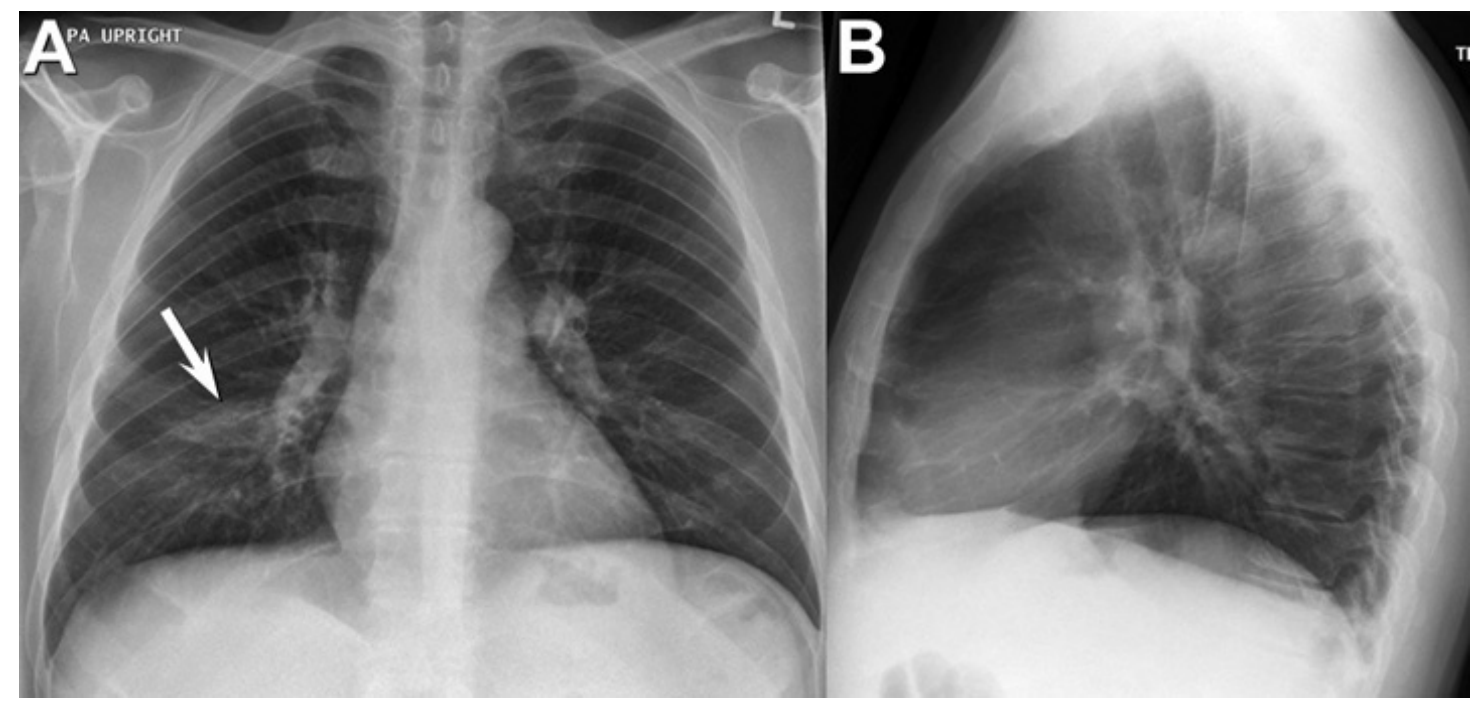

Figure 2. Frontal (A) and lateral (B) chest radiography shows a poorly defined, faintly nodular opacity at the right base (arrow), primarily seen on the frontal projection.

No evidence of peribronchial or mediastinal lymph node enlargement is seen, no are linear or reticular abnormalities. The heart size appears normal.

The patient underwent abdominal cross sectional imaging to address his complaint of weight loss and jaundice, which disclosed abnormalities of the right kidney and liver (Figure 3), with cholelithiasis and possibly some thickening of the extrahepatic bile ducts.

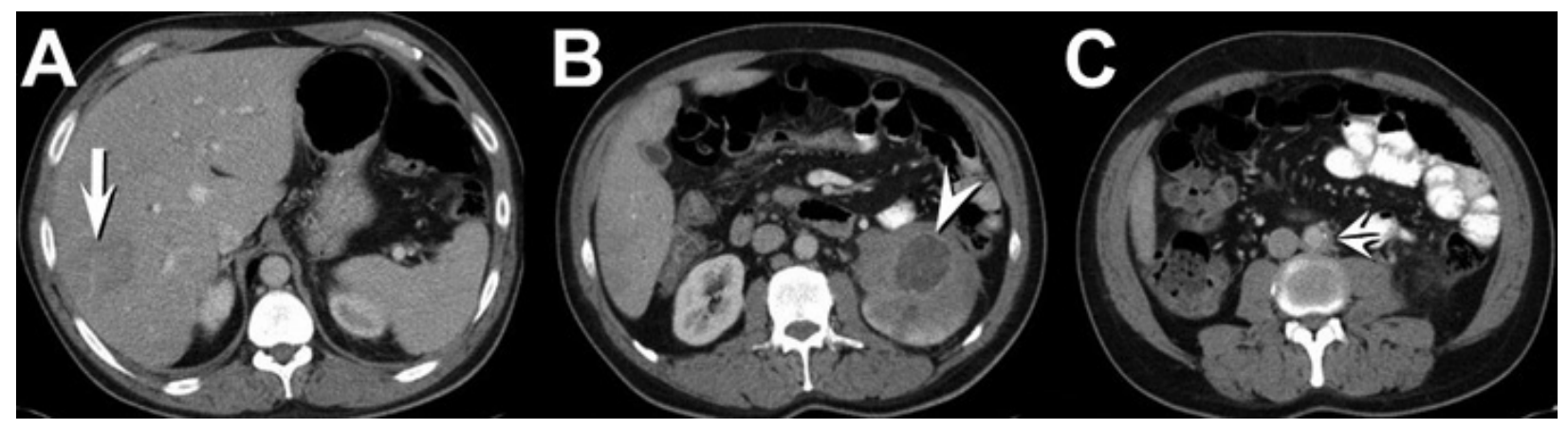

Figure 3. Representative images from axial enhanced abdominal CT showing heterogeneous wedge-shaped low attenuation in the liver (arrow), enlargement of the left kidney with a low attenuation lesion anteriorly (arrowhead) and diminished left renal enhancement, and some mild left periaortic thickening (double arrowheads). 
Which of the following would be most useful for the evaluation of this patient?

1. Contrast-enhanced thoracic CT

2. Dermatologic consultation

3. Flexible fiberoptic bronchoscopy

4. Pulmonary function testing

5. Repeat frontal chest radiography 


\section{Correct! \\ 1. Contrast-enhanced thoracic CT}

Repeat chest radiography would be of little use since as abnormalities were already noted at the presentation chest radiograph, and significant abnormalities have been detected at abdominal imaging. Obtaining pulmonary function testing could be of benefit as well, although normal results would not negate the fact that chest imaging abnormalities have been noted and require further assessment. Bronchoscopic evaluation is premature at this point, given that further non-invasive chest investigations could provide information sufficient to obviate an invasive procedure. Given that chest radiography disclosed a possible abnormality and significant abnormalities have been detected at cross sectional abdominal imaging, further evaluation with contrastenhanced thoracic CT is appropriate.

The patient underwent enhanced CT of the thorax (Figure 4).

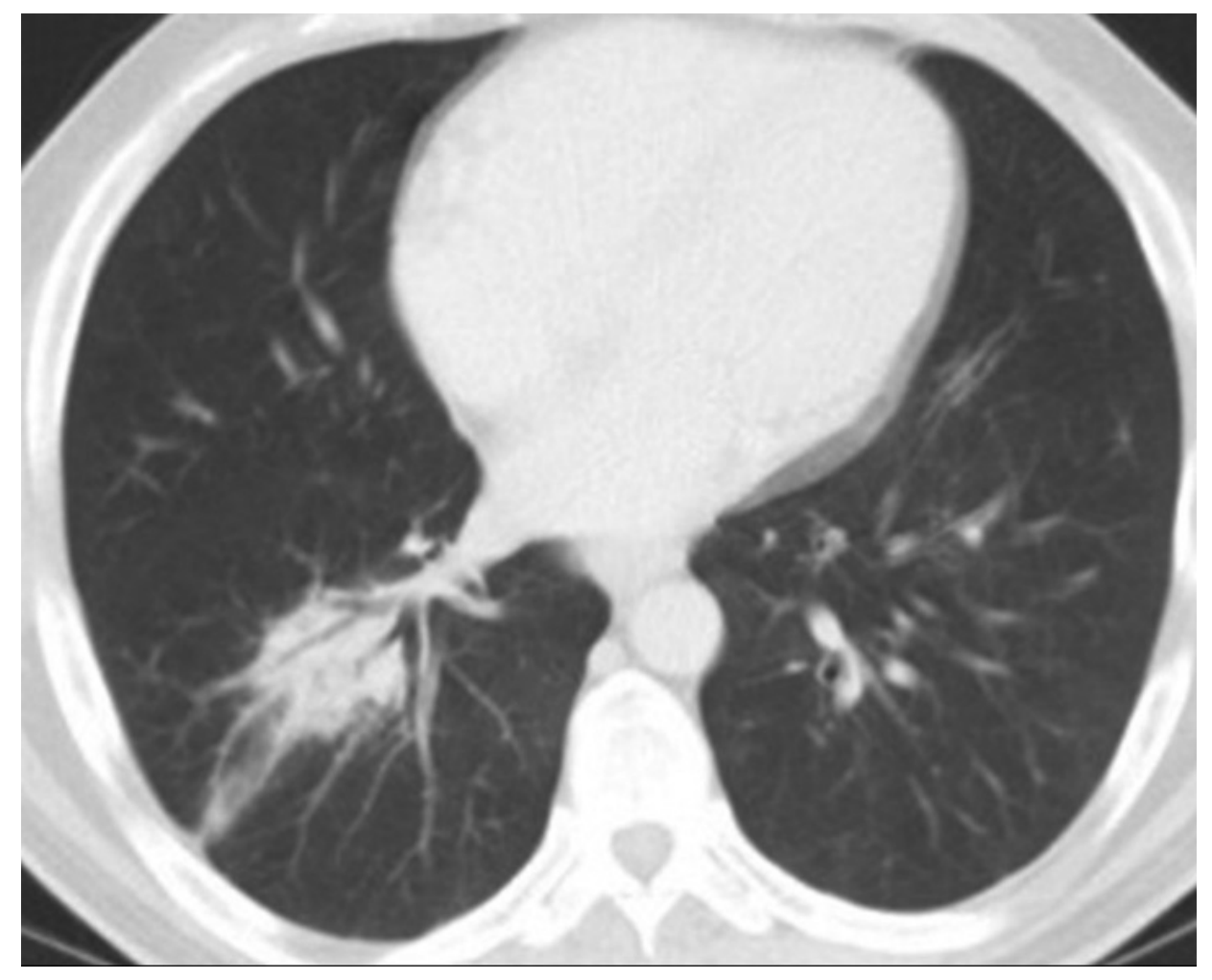

Figure 4. Representative image from axial enhanced thoracic CT.

Which of the following is correct regarding the description of the thoracic CT findings?

1. CT shows a focal nodular opacity with air bronchogram formation

2. CT shows diffuse ground-glass opacity

3. CT shows diffuse, small nodules consistent with a centrilobular distribution

4. CT shows multifocal bronchiectasis

5. CT shows multifocal reticulation suggesting fibrotic disease 


\section{CT shows a focal nodular opacity with air bronchogram formation}

Thoracic CT shows a focal, nodular opacity in the right lower lobe with several air bronchograms, correlating with the findings seen at chest radiography. The visualized airways are normal; no bronchiectasis is present. No small nodules are present, nor is diffuse ground-glass opacity seen. Three is no evidence of reticulation or linear opacity to suggest the presence of fibrotic lung disease.

The patient underwent upper endoscopy and colonoscopy and endoscopic retrograde cholangiopancreatography, which did not disclose specific abnormalities.

Which of the following is the most appropriate next step for the evaluation of this patient?

1. Endovascular biopsy of the abdominal aortic abnormality

2. Flexible fiberoptic bronchoscopy

3. Mediastinoscopy

4. Nephron-sparing surgery for the renal lesion

5. Percutaneous biopsy of the liver lesion 


\section{Correct! \\ 2. Flexible fiberoptic bronchoscopy}

Among the choices listed, flexible fiberoptic bronchoscopy probably has the best chance to obtain a tissue diagnosis for this patient. Percutaneous biopsy of the liver is a reasonable consideration, but the wedge-shape of the abnormality, with little evidence of mass effect, suggest that the finding could merely reflect focal fatty infiltration rather than a true infiltrative abnormality. A hepatic infarct could present in this fashion, but percutaneous biopsy of liver infarction may disclose hepatocyte necrosis but not a specific etiology. Nephron-sparing surgery is commonly performed for suspected localized renal malignancy. Renal malignancy is a consideration for the renal lesion in this patient, and a surgical approach would certainly provide adequate material for diagnosis, but surgery would not be appropriate in the context of multiple symptoms not directly attributable to renal malignancy in the context of a number of extra-renal lesions that still require explanation. Mediastinoscopy cannot approach the lung lesion, and no lymph node enlargement or mediastinal lesion was reported at thoracic CT, so this procedure would not be appropriate. Endovascular biopsy is technically feasible but rarely performed, and usually is performed for solid intraluminal lesions, such as suspected sarcoma.

The patient underwent percutaneous liver biopsy, which showed only fatty infiltration. Flexible fiberoptic bronchoscopy was then performed and the central airways appeared normal, but biopsy of the right lower lobe lesion revealed only non-specific inflammatory changes.

Which of the following is the most appropriate next step for the evaluation of this patient?

1. Laparotomy

2. Percutaneous biopsy of the renal lesion

3. Repeat flexible fiberoptic bronchoscopy with navigation and endobronchial ultrasound

4. Retrograde ureterography

5. Video-assisted thoracoscopic biopsy of the right lung lesion 


\section{Correct!}

\section{Percutaneous biopsy of the renal lesion}

Among the choices listed, percutaneous biopsy of the left renal lesion is the least invasive and most likely to provide a tissue diagnosis. Often percutaneous renal biopsy is performed in the context of a solid-appearing renal lesion to assess for malignancy, not infrequently in combination with percutaneous ablation techniques. In this case, percutaneous sampling solely for diagnosis would be the procedure performed. Repeating bronchoscopy would likely not be of benefit, even with the addition of navigation and endobronchial ultrasound, when the first bronchoscopic procedure adequately sampled the lesion but failed to obtain a tissue diagnosis. Retrograde ureterography can be performed to examine the renal collecting system and urothelium and obtain cytology for examination, typically for malignancy, but the renal lesion in this case is not within the collecting system, and therefore this procedure would have a reduced diagnostic yield. Video assisted thoracoscopic biopsy of the right lung lesion is technically feasible and would obtain a tissue diagnosis, but is a more aggressive approach than percutaneous biopsy of the renal lesion, or, for that matter, percutaneous transthoracic needle biopsy. Similarly, laparotomy could provide material sufficient for diagnosis if disease in the retroperitoneum that is accessible is identified, but the renal lesion is, by definition, a retroperitoneal organ and may therefore not be readily accessible through this approach.

The patient underwent percutaneous fine needle aspiration and core biopsy of the left renal mass.

Among the choices listed, which is the most likely diagnosis to explain the renal, aortic, and lung lesions?

1. IgG4 - sclerosing disease

2. Mycobacterium tuberculosis infection

3. Primary renal malignancy with pulmonary metastases

4. Renal abscess with phlebitis and septic embolization

5. Vasculitis with pulmonary, renal, and aortic involvement 


\section{Correct! \\ 1. IgG4 - sclerosing disease}

The renal lesion could be a primary malignancy- the appearance, with focal central low attenuation and foci of calcification is, however, atypical for that diagnosis. The appearance of the lung lesion is atypical for metastatic malignancy. Typically metastatic lesions are circumscribed, or, when hemorrhagic, may have poorly defined borders, but rarely will metastatic disease to the lungs present with such prominent air bronchograms. Given the low attenuation in the renal lesion, and renal abscess is a consideration, although the patient's history does not support that diagnosis. The lung lesion's appearance, however, is not suggestive of septic embolization. Septic emboli typically appear as multiple, bilateral, peripheral poorly defined opacities that fairly rapidly undergo cavitation; air bronchograms are not a typical feature of septic emboli. Furthermore, no renal vein thrombosis was noted at abdominal imaging. Vasculitis with combined pulmonary, renal, and aortic involvement is a good consideration when attempting to link pathology among these three organ systems, but the imaging appearances in this patient are not typical of vasculitis affecting these organs. Vasculitis in the lung typically appears as peripheral poorly defined nodules or masses that cavitate, or multifocal ground-glass opacity, reflecting hemorrhage; a solitary nodule or mass with air bronchograms is not suggestive of vasculitis. Vasculitis involving the aorta usually appears as concentric wall thickening, rather than the eccentric thickening seen in this patient \{although this pattern can occur with vasculitis). Finally, renal involvement with vasculitis does not typically appear as a focal mass-like lesion and is commonly bilateral, rather than unilateral. Although uncommon, IgG-4 related disease is the entity that could potentially explain the pulmonary, renal, and aortic lesions in this patient.).

The patient's renal biopsy showed storiform (resembling the spokes of a cartwheel with spindle cells radiating from a center) proliferative fibrosis with extensive inflammation, including numerous plasma cells. IgG and IgG-4 immunohistochemical stains highlighted greater than 30 IgG-4 plasma cells / high power field, with one-third of the plasma cells found to be IgG-4 positive. No glomerulonephropathy was seen. The diagnosis was therefore IgG-4 tubulo-interstitial nephritis.

The patient was considered to have IgG-4 related disease, and this disorder was thought to potentially explain all the imaging findings. The patient was begun on $40 \mathrm{mg}$ prednisone / day. The patient was re-imaged 2 months later, and regression of the biliary tract thickening, renal lesion, aortic thickening, and lung lesion (Figure 5) had either completely or nearly completely resolved. 


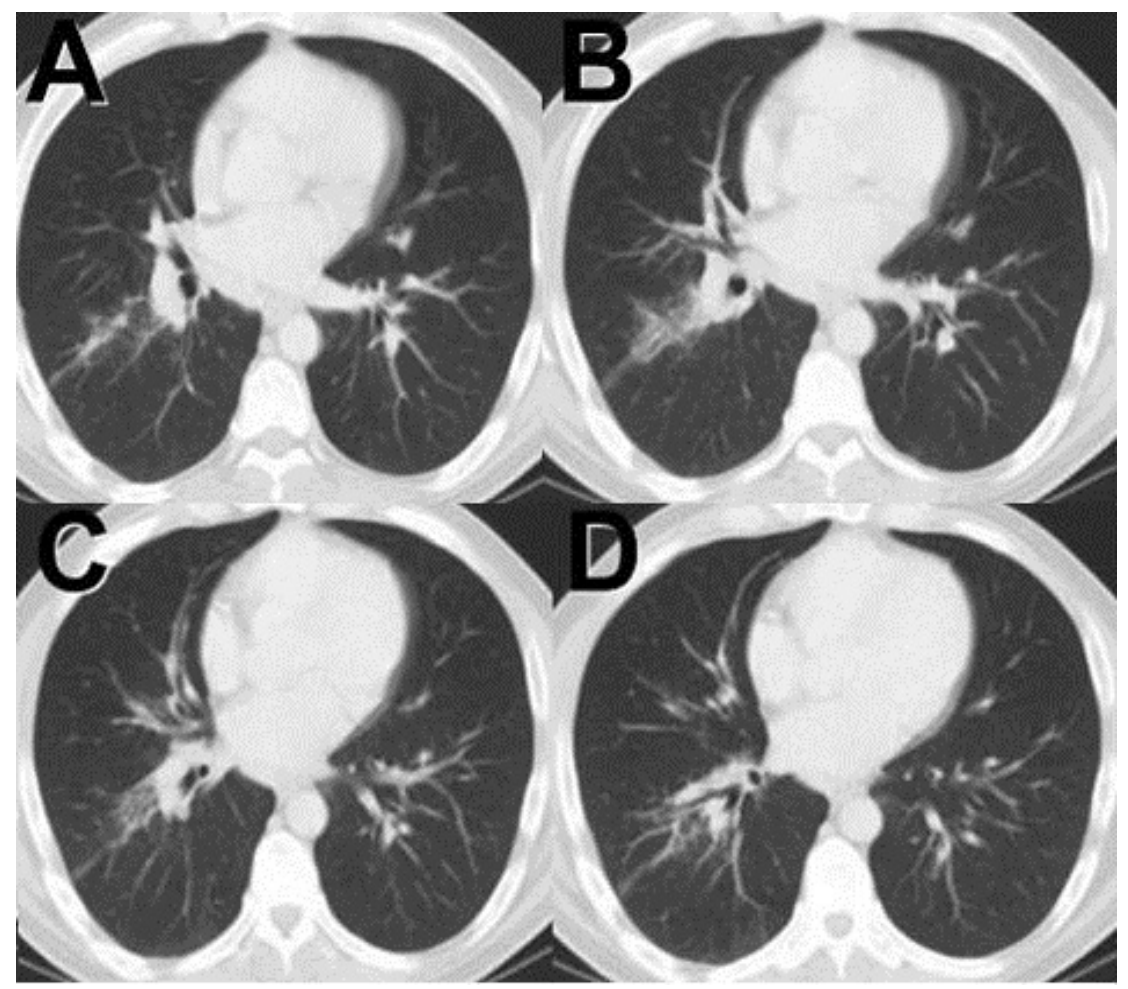

Figure 5. Representative images from the repeat thoracic CT scan 2 months after the first CT scan showing regression of the lung lesion.

Diagnosis: IgG-4 related disease

\section{References}

1. Campbell SN, Rubio E, Loschner AL. Clinical review of pulmonary manifestations of IgG4-related disease. Ann Am Thorac Soc. 2014;11(9):1466-75. [CrossRef] [PubMed]

2. Fei Y, Shi J, Lin W, Chen Y, Feng R, Wu Q, Gao X, Xu W, Zhang W, Zhang X, Zhao $Y$, Zeng $X$, Zhang F. Intrathoracic Involvements of Immunoglobulin G4-Related Sclerosing Disease. Medicine (Baltimore). 2015 Dec;94(50):e2150. [CrossRef] [PubMed]

3. Gao Y, Seidman MA, Bankier AA. Case 196: Immunoglobulin G4-related disease. Radiology. 2013; 268(2):604-609. Erratum in: Radiology 2014;270(1):314.

[CrossRef] [PubMed]

4. He Y, Du X, Ding N, Li Z, Zhou W, Chen B, Jin Z, Sun H, Zhu L, Xue H. Spectrum of IgG4-related disease on multi-detector CT: a 5-year study of a single medical center data. Abdom Imaging. 2015;40(8):3104-16. [CrossRef] [PubMed]

5. Raj R. IgG4-related lung disease. Am J Respir Crit Care Med. 2013;188(5):527-9. [CrossRef] [PubMed]

6. Raj R, Boddipalli V, Brown C, Dematte J, Raparia, K. IgG4-related lung disease. Clin Pulm Med. 2014;21:230-8. [CrossRef] 